\section{$\underset{\substack{\text { hommes } \\ \text { \& migrations }}}{ }$}

\section{Hommes \& migrations}

Revue française de référence sur les dynamiques

migratoires

\section{$1283 \mid 2010$}

Cuisines et dépendances

\title{
Lilian Thuram, Mes étoiles noires, de Lucy à Barack Obama
}

Paris, Éd. Philippe Rey

\section{Terence Carbin}

\section{(2) OpenEdition}

12 Journals

\section{Édition électronique}

URL : http://journals.openedition.org/hommesmigrations/1032

DOI : 10.4000/hommesmigrations. 1032

ISSN : 2262-3353

Éditeur

Musée national de l'histoire de l'immigration

\section{Édition imprimée}

Date de publication : 1 janvier 2010

Pagination : 206-207

ISBN : 978-2-919040-04-9

ISSN : 1142-852X

Référence électronique

Terence Carbin, "Lilian Thuram, Mes étoiles noires, de Lucy à Barack Obama », Hommes \& migrations [En ligne], 1283 | 2010, mis en ligne le 29 mai 2013, consulté le 22 septembre 2020. URL : http:// journals.openedition.org/hommesmigrations/1032; DOI : https://doi.org/10.4000/ hommesmigrations.1032

Ce document a été généré automatiquement le 22 septembre 2020.

Tous droits réservés 


\section{Lilian Thuram, Mes étoiles noires, de Lucy à Barack Obama}

Paris, Éd. Philippe Rey

Terence Carbin

\section{RÉFÉRENCE}

Lilian Thuram Mes étoiles noires, de Lucy à Barack Obama, Paris, Éd. Philippe Rey, 400

pages, 18 euros

1 Il est inconcevable aujourd'hui que l'on continue à traiter des Noirs... à la légère. Pourtant, l'enseignement de l'histoire persiste à les maltraiter en les laissant dans l'ombre. Pourquoi?

2 L'histoire des Noirs ne commence pas avec la traite et ne se réduit pas à l'esclavage. C'est une évidence pour un esprit éclairé, mais pas pour tous, surtout pour les adultes en devenir qui n'ont d'autres perspectives que les livres d'histoire, qui occultent une partie de l'humanité. Tout reste à faire...

3 C'est ce "tout reste à faire..." qui a initié ce livre de Lilian Thuram. Un "tout" frontières, un "tout" brouillard, un "tout" manipulation de l'Histoire, écrasement des esprits sous le rouleau compresseur des inhibitions et des peurs qui nourrissent le racisme et la ségrégation ; pire, une méconnaissance qui engendre frustrations, préjugés et violence contre les autres et soi-même. Donnons des perspectives nouvelles à ces enfants à la peau noire en leur montrant des héros idoines qui les mèneront à labourer le champs des possibles par identification, nous suggère Lilian Thuram qui, en multipliant les références, offre des portraits d'hommes, et de femmes - il faut le noter -, qui ont œuvré, depuis l'aube des temps, à la Grande Histoire humaine, malgré la couleur de leur peau.

4 Ce livre d'histoires singulières nous convie à entrer dans l'Histoire par l'absence l'absence dans les livres d'histoire justement - de scientifiques noirs (Firmin, Modibo Diarra, Carver, Dickinson, Spikes...), d'écrivains noirs (Césaire, Wright, Pouchkine...), de 
polytechniciens noirs (Camille Mortenol premier noir à y entrer), d'explorateurs noirs (Mathew Henson), de reines (Anne Zingha...), de philosophes (Esope, Térence, juste évoqué), de poétesses (Philis Wheatley), de résistants de la guerre de 40 (Addi Bâ), d'artistes peintres de l'académie de Rome (Guillaume Guillon Lethière) ou de musiciens classique (le plus fameux d'entre eux : le chevalier de Saint-George), qui ont tous une peau sombre et ce même désir viscéral d'être considérés simplement en être humain.

5 En face, l'homme blanc ne s'est pas privé de priver l'homme noir de son histoire. Un président de la république française s'est même autorisé à dire à Dakar en 2007 que l'africain "n'est pas assez entré dans l'histoire" et que "le paysan africains ne connait que l'éternel recommencement du temps rythmé par la répétition des mêmes gestes et des mêmes paroles. Dans cet imaginaire où tout recommence toujours, il n'y a de place ni pour l'aventure humaine ni pour l'idée de progrès".

6 Le troisième millénaire a débuté avec les mêmes réflexes que ceux des siècles précédents. Pourquoi? Par un manque de connaissance et de culture. "Le travail d'éducation n'a pas été fait", dit encore Thuram. "Si nous voulons vraiment changer notre société, lutter contre le racisme, ce n'est ni sur la discrimination positive ni sur le communautarisme qu'il faut compter. Seul le changement de nos imaginaires peut nous rapprocher et faire tomber nos barrières culturelles; là seulement nous pourrons dépasser l'obstacle majeur qui se cache derrière des mots comme 'minorité visible', 'diversité' - les 'vous' et 'nous' déterminés par la couleur de peau."

7 Sur les quarante-cinq portraits de ce livre (sans compter la longue liste des scientifiques, chercheurs et inventeurs de la page 223), certains méritent qu'on s'y arrête, tel celui, cité en titre, de Barack Obama, président des États-Unis ou celui de Pouchkine le plus grand poète russe, ou bien encore celui de Mongo Béti, l'écrivain et militant, de Jean-Jacques Dessaline, le libérateur d'Haïti, d'Anton Wilhelm Amo, le philosophe des lumières, ou même de Luther King, le pasteur du rêve. Mais insérer ceux de Mohamed Ali, le boxeur frimeur, de Tupac Shakur, le rappeur gangster et même celui de Marcus Garvey, l'apôtre du retour en Afrique, me semble contre-productif au vu du projet de l'auteur. Ceux-ci sont négrocentrés, sinon afrocentrés, et ont eu un discours, sinon une vie, à l'encontre d'un combat plus inclusif et coopératif entre les êtres d'où qu'ils soient ; l'idéologie raciste par réaction persiste dans leur approche de l'humain. "Black is beautiful" est leur crédo.

8 Un regret, un grand : l'absence d'Alexandre Dumas, le plus grand de nos écrivains. Ni le père, ni le fils, ni le génial général juste évoqué en tant que "diable noir". La panthéonisation du père des Trois Mousquetaires ne suffit pas, car, aujourd'hui encore, on ignore la couleur de peau du sieur Dumas. Et pour preuve dans un film récent, l'argent a blanchit sa peau à travers un acteur bankable.

9 Face à des siècles de ségrégation sociale et historique, pour certains, les bonnes intentions et la non-violence ne suffisent pas. La violence non plus d'ailleurs. Car, comme le dit encore Lilian Thuram: "Tant que nous resterons prisonniers de l'idéologie des scientifiques qui ont classifié les femmes et les hommes en 'supérieurs' et 'inférieurs', nous ne pourrons pas comprendre que l'âme noire, le peuple noir, la pensée noire, n'existent pas plus que l'âme banche, le peuple blanc ou la pensée blanche."

10 L'ancien footballeur international et créateur d'une fondation d'éducation contre le racisme, par ce livre simple, sans le pathos de la victimisation, contribue à offrir une histoire parallèle, une contre-histoire, celle de ceux qui, oubliés volontairement, ont 
porté les valeurs humaines au plus haut de l'humanité. Sur le socle indéboulonnable de l'admiration inhérente à l'homme, les voici étoiles admirables qui pigmentent le firmament de leur singularité pour guider quiconque souhaite sculpter sa propre statue par-delà l'épiderme. 